\title{
NON-LINEAR OPTIC POLARIZATION IN LITHIUM SULPHATE MONOHYDRATE CRYSTALS
}

\author{
Z. Czapla, M. Degsika \\ Institute of Experimental Physics, University of Wrocław \\ Pl. M. Borna 9, 50-204 Wrocław, Poland \\ E. Lebedeva And A. Pirozerski \\ Institute of Physics, Saint-Petersburg State University \\ Ulyanovskaya str. 1, 198904 Saint-Petersburg, Russia
}

(Received August 25, 2000; revised version February 5, 2001)

The "steady-state" non-linear optic polarization arising in lithium sulphate monohydrate crystals under the influence of the short pulse laser irradiation has been investigated in the temperature range of $95-305 \mathrm{~K}$. The reversal of the sign of the components of the generalised non-linear susceptibility tensor $\tilde{\chi}$ has been found at about $105 \mathrm{~K}$. The change of the photoresponse kinetics and a drastic temperature dependence of the components $\tilde{\chi}_{i j k}$ in the temperature range of $95-105 \mathrm{~K}$, as well as the change of the spectral dependence of the photoresponse were observed.

PACS numbers: 42.65.-k, 72.40.+w, 77.70.+a, 77.80.-e

\section{Introduction}

Lithium sulphate monohydrate (LSM) is a well-known pyroelectric crystal [1]. At room temperature the crystal is monoclinic and belongs to the point symmetry group 2. An interesting feature of LSM is the change of the pyroelectric coefficient sign at about $100 \mathrm{~K}$ [2-4]. A similar phenomenon was also observed in many other ferro- and pyroelectric crystals, e.g. in tourmaline (12-21 K), triglycine sulphate $(5 \mathrm{~K})$, lithium niobate $(11 \mathrm{~K})$. However, in LSM it is observed at a much higher temperature (100-110 K) [5].

Lang [3] proposed an interpretation of this sign reversal. It is well known that the pyroelectric effect at constant stress is a composition of the primary effect (i.e. pyroelectric effect at constant strain) and the piezoelectric effect due to the thermal strain. Lang calculated the primary and secondary coefficients over the temperature range of $4.2-320 \mathrm{~K}$. These coefficients cancel each other at about $106 \mathrm{~K}$, which results in a reversal of the total pyroelectric coefficient sign. 
The temperature dependences of the pyroelectric coefficient and of the spontaneous polarization have been investigated by Gavrilova et al. [4]. In this experiment an appearance of spontaneous polarization components along directions $x$ and $z$ has been observed below $110 \mathrm{~K}$. On the basis of these results a polar phase - polar phase transition connected with the alteration of water molecule state has been proposed. The neutron diffraction structural study performed at 298, 80, and $20 \mathrm{~K}$ showed the rotations and changes of the values of the dipole moments, see $[6,7]$.

To get additional information about properties of the LSM crystal we have carried out the measurements of the photoresponse due to the appearance of non-linear polarization of medium on "zero" frequency [8-13].

The generalized non-linear polarization of the second order $\pi^{0}$ can be described by the generalized non-linear susceptibility tensor $\tilde{\chi}$

$$
\pi_{i}^{0}=\varepsilon_{0} \tilde{\chi}_{i j k}^{0=\omega-\omega} E_{j}^{\omega} E_{k}^{\omega}
$$

where $E_{i}^{\omega}$ are the projections of the Fourier components of the electric field vector of the optical wave. In general, the tensor components $\tilde{\chi}_{i j k}$ are complex numbers. This tensor describes the effect of optical rectification (OR) [8, 9], Glass current (GC) $[10,11]$, excited states dipole effect (EDE) $[12,13]$.

\section{Experimental method}

LSM single crystals were grown from saturated water solution of lithium sulphate by the slow evaporation method at the constant temperature of $303 \mathrm{~K}$. The samples were cut out along the crystallophysical axes $x, y$ (polar axis), $z$.

We used a $Q$-switched YAG: $\mathrm{Nd}^{3+}$ laser as the radiation source, the wavelengths being $1.06 \mu \mathrm{m}$ (main mode), $0.53 \mu \mathrm{m}$ and $0.35 \mu \mathrm{m}$ (the second and third harmonic, respectively). The duration of the first harmonic was $15 \mathrm{~ns}$.

The samples under investigation were placed into a capacitor-type registration system. The pulse voltage appearing on capacitor during the passage of light pulse was measured. Time resolution of the registration system was about $10^{-9} \mathrm{~s}$.

The use of a cryostat allowed us to perform the measurements in the temperature range from $95 \mathrm{~K}$ to $305 \mathrm{~K}$.

\section{Results}

We have investigated kinetics of the photoresponse along axes $x, y, z$ for the wavelengths of $1.06,0.53,0.35 \mu \mathrm{m}$ at different polarizations of the laser beam and over the temperature range of $95-305 \mathrm{~K}$. At room temperature the photoresponse was observed only for $\lambda=1.06 \mu \mathrm{m}$ due to little power of higher harmonics. Its amplitude dependence on the laser beam intensity was linear up to $10^{7} \mathrm{~W} / \mathrm{cm}^{2}$.

The photoresponse along $y$ axis is represented in Fig. la for the time interval of $0-35 \mathrm{~ns}$ and in Fig. $2(0.1-4 \mu \mathrm{s})$. Its leading edge was $60 \mathrm{~ns}$, followed by the exponential decay with a characteristic time of $5 \mu \mathrm{s}$, on which the piezoelectric signal with a period of $1 \mu$ s was superimposed (see Fig. 2). The amplitude of photoresponse was slightly dependent on the laser beam polarization. The photoresponse along $x$ and $z$ axes had similar kinetics, its amplitude being nearly one-half of that in the $y$ direction. 


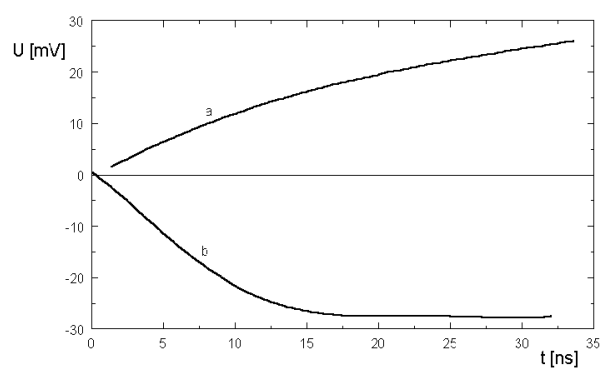

Fig. 1

Fig. 1. The photoresponse along the $y$ axis: $a-$ room temperature, $b-100 \mathrm{~K}$.

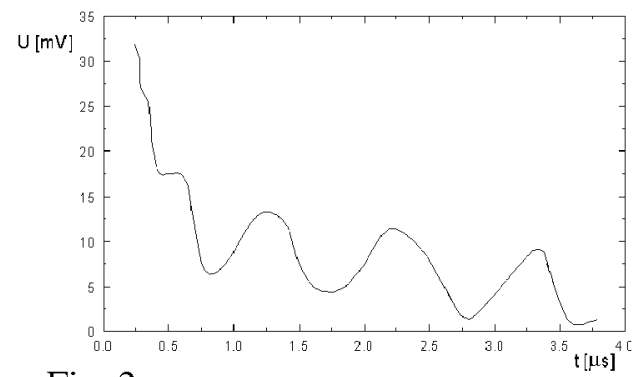

Fig. 2

Fig. 2. Piezoelectric signal.

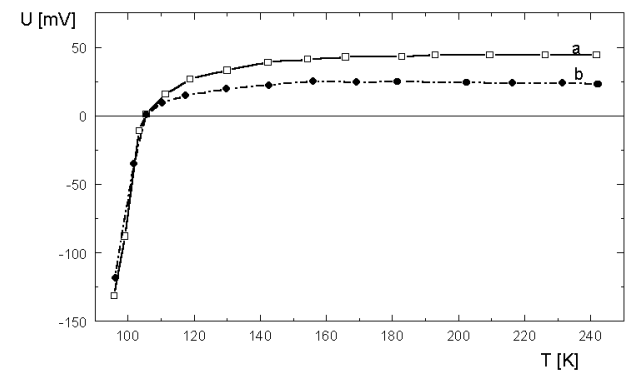

Fig. 3. The temperature dependence of the photoresponse amplitude at $\lambda=1.06 \mu \mathrm{m}$ along the $y$ - (a) and $z$-axes (b). 
The temperature dependences of the photoresponse amplitude at $\lambda=1.06 \mu \mathrm{m}$ along $y$ (Fig. 3a) and $z$ (Fig. 3b) axes were measured. It was found that the signal changed the sign at the critical temperature $T_{\mathrm{c}} \approx 105 \mathrm{~K}$, and its amplitude increased drastically below this point. At $95 \mathrm{~K}$ the effective value of $\tilde{\chi}_{222}$ was about $-5.3 \times 10^{-10} \mathrm{~m} / \mathrm{V}$ in contrast to $1.8 \times 10^{-10} \mathrm{~m} / \mathrm{V}$ at room temperature. Moreover, the photoresponse kinetics has changed (see Fig. 1), in particular, the leading edge was about $25 \mathrm{~ns}$ at $95 \mathrm{~K}$ in contrary to $60 \mathrm{~ns}$ at room temperature.

At $95 \mathrm{~K}$ the photoresponse along $y$ axis appears for $\lambda=0.53 \mu \mathrm{m}$, which indicates that the corresponding components of the non-linear susceptibility tensor are about 5 times greater at this wavelength than at $1.06 \mu \mathrm{m}$.

\section{Discussion and conclusions}

In contrast to the stationary mode of excitation, at the pulse mode various effects could give a contribution into the photoresponse: optical rectification [8, 9], Glass current [10, 11], excited states dipole effect [12, 13], pyro- and piezoelectric effects. So, the problem of separation of different contributions arises. At the same time, using the short pulse excitation mode and the investigation of the photoresponse kinetics with a high time resolution allow us to separate contributions of different effects [14].

Firstly, the piezoelectric effect arises with a delay of hundreds nanoseconds with respect to the laser pulse and its period depends on a size of the crystal. This effect is known to be due to the thermoelastic blow [15].

Secondly, although the pyroelectric coefficient changes the sign at $105 \mathrm{~K}$ [4], its temperature dependence is quite different from the temperature dependence of the photoresponse observed in our experiments. The following features also allow one to distinguish the contribution of non-linear polarization and pyroelectric effect to photoresponse:

- Coefficients, responsible for the photoresponse, increase by five times with decreasing wavelength from $1.06 \mu \mathrm{m}$ to $0.53 \mu \mathrm{m}$ (at $95 \mathrm{~K}$ ) (the pyroeffect has an opposite spectral dependence);

- The amplitude of the photoresponse depends on the laser beam polarization. The temperature dependence of the dielectric constant $\varepsilon$ obtained in [16] shows that the change of $\varepsilon$ is so insignificant that it cannot explain the photoresponse sign reversal in this temperature range.

So, the photoresponse is connected with the appearance of the non-linear polarization of the second order and can only be due to OR, GC or EDE effects. As it was mentioned above, we can describe their contribution by the generalized non-linear susceptibility tensor of the third rank $\tilde{\chi}$. For GC effects the arising of free carriers under the influence of laser radiation is necessary, which seems improbable for the used wavelengths.

A most likely appearance of the non-linear polarization is due to the excited states dipole effect related to the dipoles moments of sulphate ions and water molecules. The neutron diffraction investigations confirm the temperature change of the relative position of these dipoles [4]. This supposition is also supported 
by a correlation of the photoresponse amplitude with the presence of $\mathrm{H}$ impurity in $\mathrm{LiIO}_{3}$ crystals [17]. In contrast to the paper [17], where $\mathrm{H}$ impurities were contamination and the photoresponse amplitude was of the order of $5-10 \mathrm{mV}$, in the LSM crystals the localization of water molecules in the crystal lattice results in a much higher photoresponse amplitude.

The drastic temperature dependence of the tensor components $\tilde{\chi}_{i j k}$ in the temperature range of $95-105 \mathrm{~K}$ with the sign reversal at $T_{\mathrm{c}} \approx 105 \mathrm{~K}$, as well as the change of the kinetics and of the spectral dependence of the photoresponse, may be connected with the phase transition suggested previously in [4].

\section{References}

[1] W. Ackermann, Ann. Physik 46, 197 (1915).

[2] V.V. Gladkii, I.S. Zheludev, Kristallografiya 10, 50 (1965) [Sov. Phys. Cryst. 10, $63(1965)]$.

[3] S.B. Lang, Phys. Rev. B 4, 3603 (1971).

[4] N.D. Gavrilova, E.G. Maksimov, V.K. Novik, S.N. Drozhdin, Fiz. Tverd. Tela 27, 2597 (1985).

[5] A.B. Esengaliev, V.K. Novik, V.N. Novikov, N.D. Gavrilova, Fiz. Niz. Temp. 18, 395 (1992).

[6] J.-O. Lundgren, A. Kvick, K.M. Karppinen, R. Liminga, S.C. Abrahams, J. Chem. Phys. 80, 423 (1984).

[7] K.M. Karppinen, R. Liminga, A. Kvick, S.C. Abrahams, J. Chem. Phys. 88, 351 (1988).

[8] M. Bass, P.A. Franken, J.F. Ward, G. Weinereich, Phys. Rev. Lett. 9, 446 (1962).

[9] R. Zawodny, S. Wozniak, G. Wagnier, Opt. Commun. 130, 163 (1996).

[10] A.M. Glass, D. Von der Linde, T.J. Negran, Appl. Phys. Lett. 25, 233 (1974).

[11] A. Mazur, U. van Stevendaal, K. Buse, M. Weber, O.F. Schirmer, H. Hesse, E. Krätzig, Appl. Phys. B 65, 481 (1997).

[12] A.M. Glass, D.H. Auston, Opt. Commun. 5, 45 (1972).

[13] A. Kholkin, O. Boiarkine, N. Setter, Appl. Phys. Lett. 72, 130 (1998).

[14] P.N. Zanadvorov, E.L. Lebedeva, V.M. Moldavskaya, E.P. Kokanyan, Fiz. Tverd. Tela 30, 2015 (1988).

[15] B.N. Morozov, Yu.M. Ayvazyan, Kvantovaya Elektronika 7, 5 (1980).

[16] M. Frindi, M. Peyrard, M. Remoissenet, J. Phys. C, Solid State Phys. 13, 3493 (1980).

[17] E.L. Lebedeva, S.A. Normatov, E.V. Charnaya, Fiz. Tverd. Tela 34, 3006 (1992). 